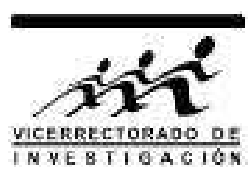

\title{
Transporte electrónico en el grafeno con nanoporos y la presencia de moléculas
}

\author{
A. P. Aslla ${ }^{* 1,2}$ y P. H. Rivera ${ }^{1}$ \\ ${ }^{1}$ Facultad de Ciencias Físicas, Universidad Nacional Mayor de San Marcos, Lima, Perú \\ ${ }^{2}$ Universidad Nacional Tecnológica del Cono Sur de Lima, Lima, Peru
}

Recibido 20 enero 2014 -Aceptado 15 abril 2014

\begin{abstract}
Analizamos el transporte electrónico en un nanodispositivo de grafeno con un nanoporo rectangular en la que se encuentran moléculas de diferentes configuraciones en el centro del nanoporo. El comportamiento de los electrones es descrito en la aproximación tight-binding y el transporte en el régimen balístico por medio de la fórmula de Landauer, donde la conductancia eléctrica es calculada a través del coeficiente de transmisión que se determina utilizando las funciones de Green retardadas de no-equilibrio. Consideramos que el nanodispositivo se encuentra conectada a dos contactos semi-infinitos también de grafeno. Los resultados muestran que la presencia de las moléculas en el nanoporo produce unas resonancias (antiresonancias) en el perfil de la diferencia del coeficiente de transmisión a través del nanodispositivo con y sin la presencia de las moléculas en el nanoporo. Estas resonancia (antiresonancias) se deben al acoplamiento de los autotestados discretos de las moléculas y los autoestados contínuos del nanodispositivo conocidos como resonancias de Fano.

Palabras claves: Grafeno, transporte cuántico, transporte balístico, funciones de Green, fórmula de Landauer, nanoporos.
\end{abstract}

\section{Electron transport in graphene with nanopore and the presence of molecules}

We analyze the electronic transport in a graphene nanodevice with a rectangular nanopore in the presence of different configurations molecules at the nanopore's center. The behaviour of the electrons is described by the tight-binding method and the transport in the ballistic regime by the Landauer's formula, where the electrical conductance is given by the transmission coefficient, which is determined using the non-equilibrium Green's functions. The nanodevice is connected to two graphene semi-infinite contacts. The results show that the presence of molecules at the nanopore center produce resonance (antiresonance) in the profile of the transmission coeficient difference between nanodevice with and without molecule at the nanopore center. These resonances (antiresonances) are due to the coupling of the molecule discrete states and the continuum states of nanodevice also called as the Fano resonances.

Keywords: Graphene, quantum transport, ballistic transport, Green functions, Landauer formula, nanopores.

El grafeno es una monocapa bidimensional conformada por átomos de carbono distribuídos en una red cristalina hexagonal. Desde su obtención experimental en el año 2004 por el grupo de Geim en la Universidad de Manchester [1], el grafeno ha mostrado unas propiedades mecánicas, térmicas y electrónicas excepcionales en sus diferentes formas tales como monocapas, bicapas, puntos cuánticos, nanoribbons, superredes y heteroestructuras con otros materiales. Las propiedades peculiares del transporte de carga en el grafeno se debe a que la estructura electrónica en los puntos de simetría K y K', llamados como los puntos de Dirac, presenta una intersección entre las bandas de valencia y conducción. Muy cerca a estos puntos las ban- das tienen una dependencia lineal respecto a la magnitud del vector de onda que permite a los electrones moverse con velocidades $v_{F} \sim 10^{6} \mathrm{~m} / \mathrm{s}$ [2], valor que se considera relativístico pues representa el $0.3 \%$ de la velocidad de la luz.

Uno de los sistemas construídos con el grafeno y que muestra cierto interés en la comunidad es la que posee un nanoporo en el medio de una cinta de grafeno. La conductancia de la cinta que incluye a un nanoporo tiene un perfil definido que depende de las dimensiones de la cinta y del tamaño del nanoporo [3. Al colocar una molécula en medio del nanoporo, la conductancia varía de acuerdo a la configuración atómica de la molécula. Una de las posibles

*aaslla@untecs.edu.pe 
aplicaciones que se visualiza respecto a este nanodispositivo es el secuenciamiento de las bases del ADN [3] y otras moléculas de interés como las proteínas.

El secuenciamiento molecular del ADN usando nanoporos ofrecería relativa ventaja respecto a los métodos bioquímicos por sensar directamente la configuración atómica de las moléculas a un costo relativamente bajo comparado con los complejos procesos bioquímicos de crecimiento y clonado. Varios grupos han reportado algunas características del transporte de carga de las moléculas del ADN, y se encuentra que cada nucleótido produce una relación corriente-voltage característico para cada base [4-7]. Además, los resultados muestran que los mecanismos de transporte de carga a lo largo del esqueleto conformado por los azúcares de desoxirribosa unidos por los grupos fosfatos son diferentes al transporte de carga entre cada par de bases unidos por dos pares de hidrógeno en la dirección transversal al esqueleto del ADN.

La idea de utilizar nanoporos de grafeno en el proceso de análisis de moléculas y la secuenciación del ADN es medir los perfiles de la conductancia del nanoporo y observar las pequeñas variaciones que sufre el perfil del conductancia cuando una molécula o un nucleótido de ADN se encuentra en el centro del nanoporo, dichas variaciones del perfil de la conductancia son característicos de la conformación atómica de la molécula y permiten, por tanto, una lectura rápida y directa del tipo de molécula que se encuentra en el centro del nanoporo. En la actualidad se viene trabajando fuertemente en el desarrollo de técnicas de medición de corriente que sean sensibles a los pequeños cambios de corriente originados por las moléculas y que permitan la producción comercial de los dispositivos de secuenciamiento de ADN.

En el presente trabajo, que es la continuación de un primer trabajo [3], simulamos el transporte de carga a través de un nanodispositivo de grafeno con un poro en el medio conectado a dos contactos también de grafeno. Calculamos la conductancia del nanodispositivo en la presencia de una molécula en el nanoporo para diferentes configuraciones de una molécula básica.

\section{Teoría}

Nuestro sistema es un dispositivo de grafeno de dimensiones $X \times Y$ en el cual se perfora un poro de dimensiones $D \times W$. La configuración electrónica de los átomos de carbono que conforman el dispositivo y los contactos son analizados por el método tight-binding considerando una interacción a primeros vecinos de los orbitales $p$ que se encuentran perpendiculares a los orbitales hibrizados $s p^{2}$ que conforman la red hexagonal. El origen de la banda de valencia y conducción del grafeno que se cruzan en los puntos $\mathrm{K}$ y $\mathrm{K}^{\prime}$ se debe a estos orbitales $p$.

El diagrama esquemático de un nanoporo rectangular de grafeno conectado a dos contactos semi-infinitos se muestra en la figura [1 donde la energía de los portadores en los contactos izquierdo y derecho están definidos por los potenciales químicos $\mu_{L}$ y $\mu_{R}$, respectivamente. El efecto de los contactos sobre el dispositivo se produce a través de las auto-energías, el transporte electrónico en el régimen balístico se analiza mediante la formula de Landauer, en la que el coeficiente de transporte se determina mediante las funciones de Green de no equilibrio (non-equilibrium Green's function).

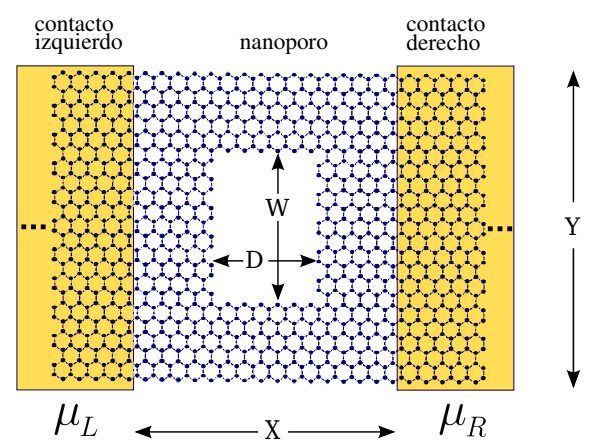

Figura 1: Representación esquemática de un nanoporo rectangular en un dispositivo de grafeno conectado a dos contactos semi-infinitos de borde zigzag.

La estructura cristalina del grafeno es descrito por dos subredes de Bravais triangulares, $A$ y $B$, mientras que la estructura electrónica donde el transporte electrónico ocurre está conformado por los orbitales acoplados tipo $p$ perpendiculares al plano del grafeno conformado por las dos redes triangulares. Las autoestados de energía de los orbitales tipo $p$ proyectados sobre los autoestados de posición en cada en cada punto de las redes triangulares son designados como $\left|\phi_{j}^{A}\right\rangle$ y $\left|\phi_{i}^{B}\right\rangle$, de modo que el hamiltoniano [89], de interacción entre los primeros sitios correspondientes a las sub-redes A y B están dados por

$$
H=-t \sum_{\langle i, j\rangle}\left|\varphi_{j}^{A}\right\rangle\left\langle\varphi_{i}^{B}\right|+\text { h.c. },
$$

donde hemos considerado que la energía de los orbitales tipo $p$ en los sitios es cero y $t=2.78 \mathrm{eV}$ es el parámetro de hopping entre primeros vecinos y $\langle i, j\rangle$ indica que la suma se efectúa sobre los primeros vecinos más próximos. Por tanto, los autovalores de energía asociados a la Ec.(1) están dados por [10]

$$
E_{k}= \pm \sqrt{1+4 \cos \left(\frac{\sqrt{3}}{2} k_{x} a\right) \cos \left(\frac{3}{2} k_{y} a\right)+4 \cos ^{2}\left(\frac{\sqrt{3}}{2} k_{x} a\right)} .
$$


Dichos autovalores graficados en tres dimensiones nos muestra que los puntos de Dirac K y K' son aquellos puntos del espacio recíproco en los cuales la Ec.(2) es igual a cero, es decir, puntos donde las bandas de conducción y de valencia se intersectan y dicho sea de paso alrededor de tales puntos $E_{k} \approx \pm \hbar v_{F}|\boldsymbol{k}|$ osea, una dependencia lineal respecto a $|\boldsymbol{k}|$.

Para describir el transporte electrónico en los nanodispositivos de grafeno consideramos el régimen balístico a temperatura $T \approx 0 \mathrm{~K}$, donde se utiliza la fórmula de Landauer para determinar la conductancia $G$ del nanodispositivo,

$$
G=\frac{2 e^{2}}{h} T(E),
$$

donde $T(E)$ es el coeficiente de transmisión a través de todos los canales permitidos del nanodispositivo próximos al potencial químico $\mu$ de los contactos, e es la carga del electrón, $h$ es la constante de Planck y 2 representa la degeneración de espín por cada canal. Para calcular $T(E)$ utilizamos las funciones de Green retardadas de no equilibrio del nanodispositivo y la información incorporada a lo largo de los contactos a través de las autoenergías [9]. Para el sistema de la figura [1 la función de Green retardada $G$ del nanodispositivo se define como

$$
G=\left[E I-H_{D}-\Sigma_{L}-\Sigma_{R}\right]^{-1}
$$

donde $I$ es la matriz identidad, $H_{D}$ el hamiltoniano del nanodispositivo y $\Sigma_{L, R}$ son las autoenergías asociadas a los contactos izquierdo $(L)$ y derecho $(R)$, respectivamente. Las autoenergías son dadas por $\Sigma_{q}=H_{D q} g_{q} H_{q D}$, donde $g_{q}$ son las funciones de Green de los contactos y $H_{q D}$ es la matriz de acoplamiento entre el nanodispositivo y los contactos $(q=L, R)$. Para determinar las funciones de Green de los contactos, $g_{q}$, utilizamos el método recursivo de convergencia rápida [11,12]. El coeficiente de transmisión $T(E)$ se expresa a través de la función de Green del dispositivo $G$ como

$$
T(E)=\operatorname{Tr}\left[\Gamma_{L} G \Gamma_{R} G^{\dagger}\right],
$$

donde $\Gamma_{L, R}=i\left(\Sigma_{L, R}-\Sigma_{L, R}^{\dagger}\right)$ expresa el acoplamiento del nanodispositivo con los contactos.

La corriente eléctrica que pasa a través del nanodispositivo a la temperatura aproximada de $0 \mathrm{~K}$ se expresa como

$$
I=\frac{2 e^{2}}{h} V \int_{\mu_{L}}^{\mu_{R}} T(E) d E,
$$

donde $|e| V=\mu_{R}-\mu_{L}$.

Por otro lado, la densidad de estados (DOS), que describe la cantidad de estados accesibles por unidad de energía se obtiene como

$$
\rho(E)=-\frac{1}{\pi} \operatorname{Tr}[\operatorname{Im}[G(E)]] .
$$

\section{Resultados y discusión}

La conductancia de un nanodispositivo adherido a contactos metálicos se determina mediante la fórmula de Landauer en la aproximación del régimen balístico. La presencia de un nanoporo en el nanodispositivo afecta la conductancia.

Aquí presentamos los resultados del coeficiente de transmisión $T(E)$ obtenidos de acuerdo a la Ec.(5) para un nanoporo rectangular $D \times W$ construído en una cinta de grafeno de dimensiones $X \times Y$ mostrados en la figura 11 Para efectos del análisis, consideramos $X=15 \sqrt{a}$ y $Y=26 a$, siendo $a=1.42 \AA$ la distancia entre cada par de átomos de carbono formando la red de panal de abeja del grafeno, como las dimensiones consideradas $X$ e $Y$ son pequeñas, éstas permiten observar eficientemente los efectos de la cuantización espacial en la conductancia, la cuantización espacial limita el número de canales de transporte electrónico permitidos y depende del ancho $Y$, por razones de tiempo computacional la dimensión longitudinal $X$ es menor que $Y$, pero el número de canales permitidos de tunelamiento es independiente de $X$.

En la figura 2 ta presentamos el coeficiente de transmisión en función de la energía correspondiente a la red finita de grafeno de dimensiones $X \times Y$ sin el nanoporo, resultado que coincide con el coeficiente de transmisión en función de la energía de los portadores de carga en nanoribbons de grafeno con borde zigzag y ancho $Y$ [13, 14]. Asimismo, en la figura 2-b se observa que la DOS en función de la energía presenta picos que coinciden con los saltos en la escalera del coeficiente de transmisión y por ende en la conductancia eléctrica de acuerdo a la Ec.(3) debido a la presencia de las singularidades de van Hove, en las que la DOS tiende a infinito. El nanodispositivo con $Y=26 a$ de borde zigzag es conductor debido a que no presenta un gap de energía entre las bandas de conducción y valencia, hecho que se traduce en el valor del coeficiente de transmisión igual a 1 en las proximidades de $E=0 \mathrm{eV}$.

En las figuras 2 $\mathrm{rc}, \mathrm{e}, \mathrm{g}$, presentamos los coeficientes de transmisión del nanodispositivo de grafeno con nanoporos de diferentes dimensiones $D \times W$, siendo $D=7 \sqrt{3} a$ y $W=4 a, 7 a$ y $10 a$, respectivamente. Los resultados muestran que la presencia de los nanoporos provocan la pérdida de la cuantización en números enteros del coeficiente de transmisión porque disminuye el número de los canales de tunelamiento, por tanto el coeficiente de transmisión total es menor que el coeficiente de transmisión del nanodispositivo de ancho $Y$. La disminución de $T(E)$ se acentúa a medida que el ancho $W$ del nanoporo se incrementa, se observa también que el $T(E)$ no es afectada por la longitud $D$ del nanoporo, afirmación que es coherente con las condiciones de transporte balístico. Los resultados mostrados en las figuras 2 - $d, f, h$ muestran que la DOS disminuye respecto a la DOS de la figura 2 a, principalmente en aquellos valores de energía donde se presentan las sin- 
gularidades de van Hove, esta disminución se debe a que la cantidad de estados permitidos en la presencia del nanoporo son menores a la cantidad de estados permitidos sin el nanoporo.

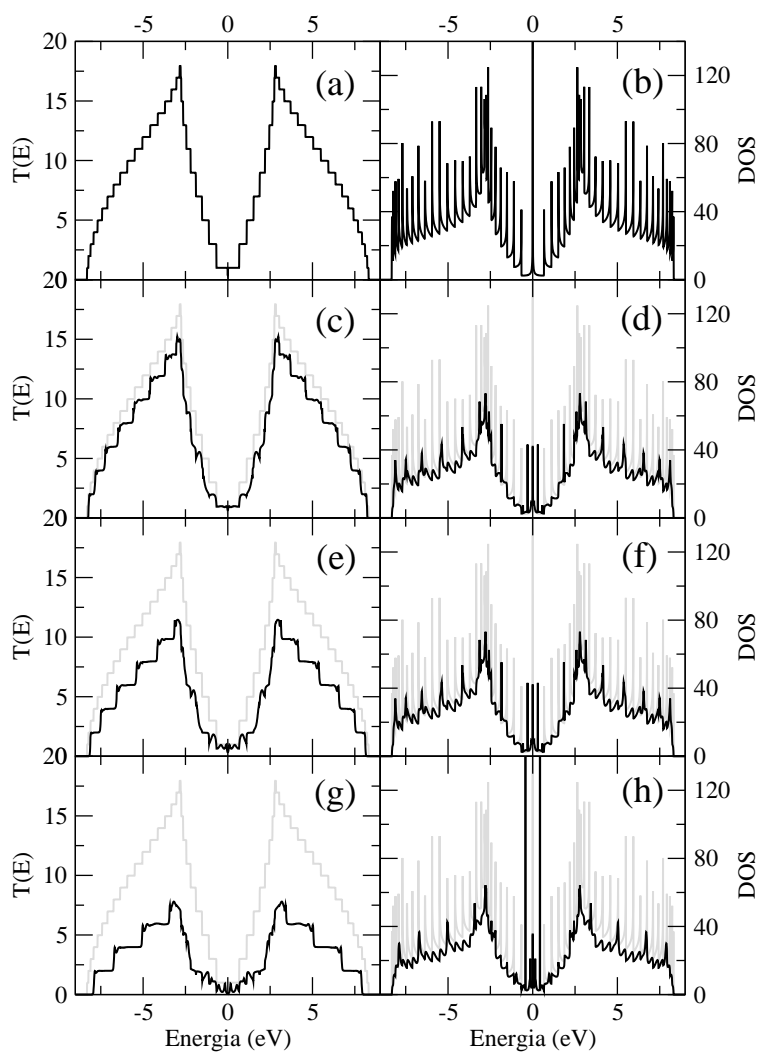

Figura 2: Se muestran los coeficientes de transmisión y la densidad de estados (DOS) para nanodispositivos de grafeno conectados a dos contactos con $\mu_{L}$ y $\mu_{R}$. Sin nanoporo (a) y (b). Con nanoporos: (c) y (d) $D=7 \sqrt{3} a$ y $W=4 a$; (e) y (f) $D=7 \sqrt{3} a$ y $W=7 a$; (g) y (h) $D=7 \sqrt{3} a$ y $W=10 a$.

A continuación dentro del nanoporo de dimensiones $D=7 \sqrt{3} a$ y $W=7 a$, cuyos coeficientes de transmisión y DOS son mostrados en la figura 2. c y d, respectivamente, colocamos diferentes moléculas con las configuraciones espaciales mostradas en la figura 3 La interacción entre los átomos de la molécula se considera en la aproximación tight-binding, de modo que el hamiltoniano que describe la molécula es $H_{m}=-\sum t_{m}[|i\rangle\langle i+1|+| i+1\rangle\langle i|]$ siendo $t_{m}$ el parámetro de hopping entre los átomos de la molécula y $|i\rangle$ representa los autoestados de energía proyectados en el espacio del operador de posición. La interacción entre el nanodispositivo y la molécula se asume también dentro de la aproximación tight-binding, teniendo en cuenta que cada átomo de la molécula interactúa con el sitio más próximo del nanodispositivo por medio del parámetro de hopping $t_{m p}$ dado por

$$
t_{m p}=-\frac{\hbar^{2}}{2 m b^{2}}=-\frac{\hbar^{2}}{2 m(r a)^{2}}=\frac{t}{r^{2}},
$$

siendo $b=r a$ la distancia entre el átomo de la molécula y el sitio que corresponde al borde del nanodispositivo más próximo.

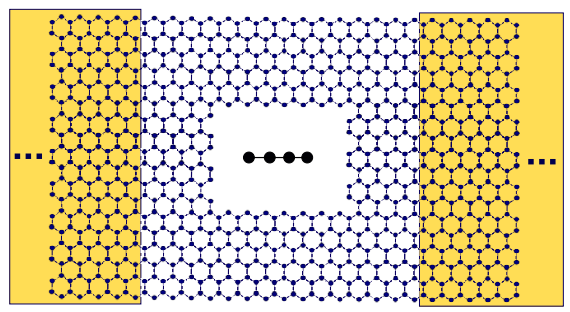

Figura 3: Representación gráfica de una molécula lineal con cuatro átomos en el centro de un nanoporo rectangular en un nanodispositivo de grafeno.

Los resultados encontrados para el coeficiente de transmisión del nanoporo de la figura 3 con una molécula lineal compuesta por cuatro átomos idénticos, que interactúan entre sí con $t_{m}=1.2 t$ se muestra en la figura 4 en la que las curvas negras representan al coeficiente de transmisión del nanodispositivo con nanoporo y la presencia de la molécula y las curvas azules representan el coeficiente de transmisión del nanodispositivo con nanoporo sin la presencia de la molécula. La gráfica muestra que existen cuatro valores de energía en las cuales $T(E)$ presenta antiresonancias, éstas ocurren para los valores de energía $E= \pm 2.05 \mathrm{eV}$ y $\pm 5.34 \mathrm{eV}$.

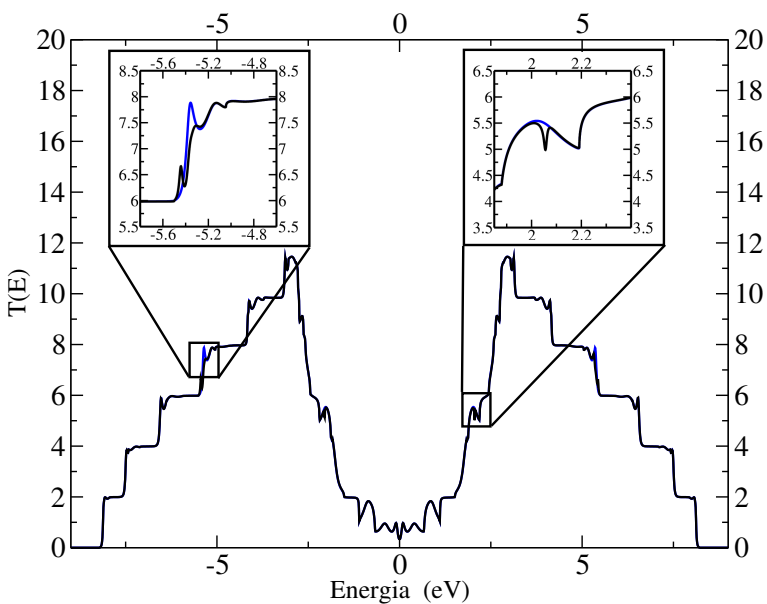

Figura 4: Coeficiente de transmisión de un nanodispositivo de grafeno con un nanoporo rectangular en la que se encuentra una molécula lineal de cuatro átomos de la figura 3 .

Debido a que las variaciones de $T(E)$ observadas en la figura 4 son pequeñas calculamos las variaciones $\Delta T$ 
del coeficiente de transmisión de un nanodispositivo con un nanoporo de dimensiones $D=7 \sqrt{3} a$ y $W=7 a$ con y sin la presencia de una molécula en el nanoporo. En estas gráficas se observan que para algunos valores de energía la diferencia $\Delta T$ son positivos indicando que la presencia de la molécula produce resonancias en el coeficiente de transmisión y los valores negativos indican que las moléculas producen unas supresiones resonantes o antiresonancias en el coeficiente de transmisión. Los resultados mostrados en la figura 5 representan $\Delta T$ en función de la energía cuando en el centro del nanoporo existen moléculas lineales compuestas de distintos números de átomos, los átomos de la molécula se consideran idénticos e interactúan entre sí con un parámetro de hopping $t_{m}=t$. En la figura 5 -a la molécula lineal es formada por tres átomos idénticos, los mismos que producen tres resonancias (antiresonancias) en $\Delta T$ en $E / t=0$ y \pm 3.9 ; cuando la molécula lineal es formada por cuatro átomos idénticos se presentan cuatro resonancias (antiresonancias) en $\Delta T$ mostrados en la figura 5.b para las energías $E / t= \pm 1.8$ y \pm 4.5 ; mientras que, si el número de átomos son cinco existen cinco resonancias (antiresonancias) en $\Delta T$ dados en la figura 5 . c para las energías $E / t=0.0 ; \pm 2.8$ y \pm 4.8 y finalmente, si el número de átomos son seis, las resonancias (antiresonancias) en $\Delta T$ son seis que ocurren para las energias $E / t= \pm 0.98 ; \pm 2.78$ y \pm 4.0 mostrados en la figura 5 . d. Estos resultados indican que la cantidad de resonancias (antiresonancias) en $\Delta T$ corresponden exactamente al número de átomos que constituyen la molécula, además la forma de las resonancias (antiresonancias) sugieren que se produce los acoplamientos entre autoestados discretos de las moléculas y los autoestados contínuos del nanodispositivo que producen las resonancias de Fano [16].
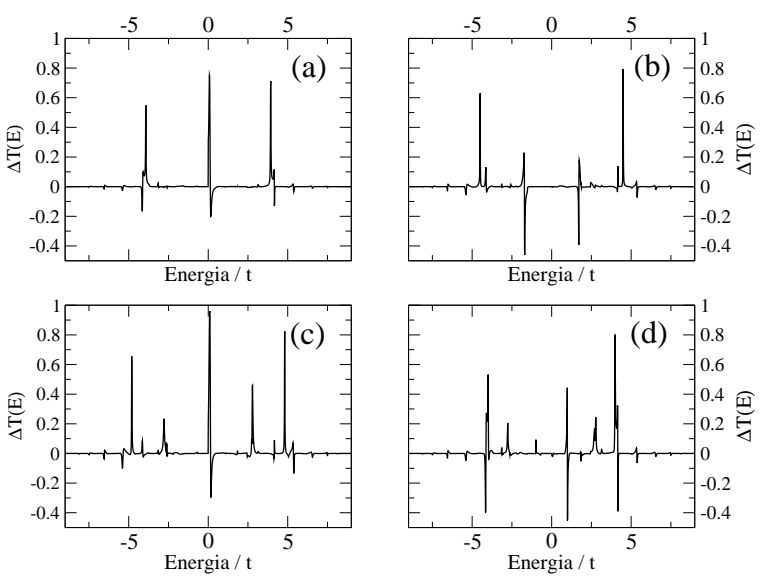

Figura 5: Variación del coeficiente de transmisión $\Delta T$ de nanodispositivos de grafeno con nanoporos rectangulares en la que se encuentran moléculas lineales formadas por diferente número de átomos identicos (a) 3 átomos, (b) 4 átomos, (c) 5 átomos y (d) 6 átomos.
Con la finalidad de emular el efecto de moléculas compuestas por diferentes tipos de átomos, consideramos una molécula lineal formada por cuatro átomos, que interactúan entre sí con diferentes parámetros de hopping $t_{m}$. En la figura 6-a consideramos $t_{m}=0.6 t$ ocurriendo las resonancias (antiresonancias) de $\Delta T$ en $E / t= \pm 0.7$ y \pm 1.8 , cuando $t_{m}=0.8 t$ las resonancias (antiresonancias) ocurren para $E / t= \pm 1.0$ y 2.7 , figura [6 b, si $t_{m}=1.2 t$ las resonancias (antiresonancias) en $\Delta T$ ocurren en $E / t=$ \pm 2.0 y \pm 5.4 mostrados en la figura 6 c y si $t_{m}=1.4 t$ las resonancias (antirersonancias) en $\Delta T$ se muestran en la figura 6 6 d y ocurren para las energías $E / t= \pm 2.5$ y \pm 6.4 . De acuerdo a estos resultados inferimos que las moléculas lineales constituídas por diferentes tipos de átomos producen resonancias (antiresonancias) en $\Delta T$ que muestran características diferenciadas para cada tipo de átomo en las moléculas.
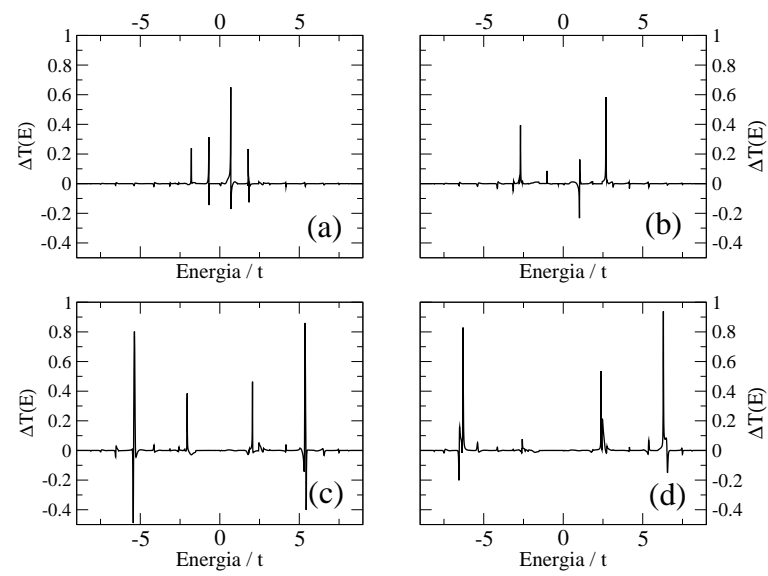

Figura 6: Variación del coeficiente de transmisión $\Delta T$ de un nanodispositivo de grafeno con un nanoporo rectangular en la que se encuentra una molécula lineal de cuatro átomos con distintos parámetros de hopping $t_{m}$. (a) $t_{m}=0.6 t$, (b) $t_{m}=0.8 t$, (c) $t_{m}=1.2 t$ y (d) $t_{m}=1.4 t$.

Finalmente, para simular moléculas con diferentes estructuras tal como se muestran en la figura 7 consideramos que los átomos que conforman la moléculas interactúan entre sí con un parámetro de hopping $t_{m}=t$ y que se encuentran en el centro de un nanoporo rectangular de dimensiones $D=7 \sqrt{3} a$ y $W=7 a$, los perfiles de $\Delta T$ calculados se muestran en la figura 8 , en los que se observan que las resonancias (antiresonancias) en $\Delta T$ ocurren para diferentes valores de energía. Asimismo, se observa que el número de resonancias (antiresonancias) no coincide con el número de átomos que constituyen la molécula esto significa que existen algunos autoestados moleculares degenerados. 


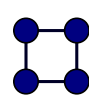

(a)

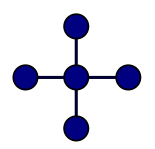

(b)

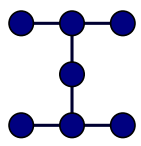

(c)

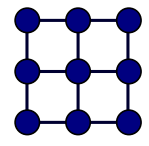

(d)
Figura 7: Tipos de moléculas que consideramos en el centro del nanoporo.
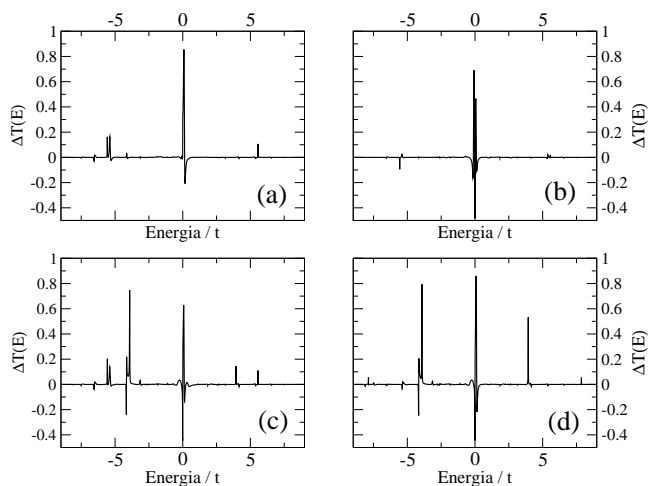

Figura 8: Variación del coeficiente de transmisión $\Delta T$ de nanoporo rectangular de grafeno con presencia de moléculas de distintos tipos mostrados en la figura 7 respectivamente.

\section{Conclusiones}

El efecto de un nanoporo en una red finita de grafeno es reducir el transporte de carga. Esto es una consecuencia de la disminución del número de estados cuánticos permitidos en el grafeno por la presencia del nanoporo que se traduce en la reducción de la conductancia eléctrica y del coeficiente de transmisión. La reducción del transporte electrónico se acentúa más cuando el ancho del nanoporo se incrementa, asimismo, debido a las condiciones de transporte balístico la longitud del nanoporo no afecta al transporte de cargas.
Las moléculas presentes en el centro del nanoporo rectangular producen resonancias y antiresonancias en el espectro de $\Delta T$ que son característicos para cada tipo de molécula, los valores de energía para los cuales ocurren dichas resonancias coinciden con las energías de los estados moleculares acoplados que cuando no poseen estados degenerados coinciden con el número de átomos que constituyen la molécula y dependen de la forma en el cual se distribuyen los átomos en la molécula y del tipo de átomo. En particular para moléculas lineales el número de resonancias (antiresonancias) de $\Delta T$ coincide exactamente con el número de átomos constituyentes de la molécula, y varía con el tipo de átomos que forman la molécula.

Para moléculas con átomos distribuídos espacialmente existe la posibilidad de la formación de estados degenerados que tienen un mismo autovalor de energía, estos presentan un espectro de $\Delta T$ muy particular y el número de resonancias (antiresonancias) es menor que el número de átomos en la molécula.

Estas particularidades que presentan los espectros de $\Delta T$ y concentrándose en determinadas regiones de energía próximos a las diferencias de los potenciales químicos abre la posibilidad de utilizar los nanodispositivos de grafeno con nanoporos como una técnica espectroscópica de cargas y corrientes para el análisis conformacional de las macromoléculas como las proteínas, para tal fin se requiere construir una base de datos de cambios en la conductancia eléctrica o el coeficiente de transmisión producida por las diferentes moléculas que conforman tales proteínas.

\section{Agradecimientos}

A. P. A. agradece a la "Asociación Familia Zuñiga y Rivero" por el apoyo económico para la redacción de la tesis de maestría mediante el Programa de Becas para Tesis de Maestría en la Facultad de Ciencias Físicas de la UNMSM. P. H. R. agradece al Consejo Superior de Investigaciones el apoyo financiero mediante los proyectos $\mathrm{N}^{\mathbf{o}} \mathrm{s}$. 111301041,121301071 y 131301081.

\section{Referencias}

[1] K. S. Novoselov, A. K. Geim, S. V. Morozov, D. Jiang, Y. Zhang, S. V. Dubonos, I. V. Grigorieva y A. A. Firsov; Science 306, 666 (2004).

[2] K. S. Novoselov, D. Jiang, F. Schedin, T. J. Booth, V. V. Khotkevich, S. V. Morozov y A. K. Geim; Nature 438, 197, (2005).

[3] A. P. Aslla y P. H. Rivera; Rev. Inv. Phys. 16, 131602101 (2013).

[4] G. F. Schneider, S. W. Kowalczyk, V. E. Calado, G.
Pandraud, H. W. Zandbergen, L. M. K. Vandersypen y C. Dekker; Nano Lett. 10, 3163 (2010).

[5] G. F. Schneider y C. Dekker; Nature Biotechnology 4, 326 (2012).

[6] K. K. Saha, M. Drndić y B. K. Nikolić; Nano Lett. 12, 50 (2012).

[7] G. Sigalov, J. Comer, G. Timp y A. Aksimentiev; Nano Lett. 8, 56 (2008).

[8] C. Bena y G. Montambaux; New Journal of Physics 11, 123-125 (2009). 
[9] S. Datta; Electronic Transport in Mesoscopic Systems; Primera edición, Cambridge University Press, New York, (1999).

[10] P. R. Wallace, Phys. Rev. 71, 622 B (1947).

[11] M. P. L. Sancho, J. M. L. Sancho y J. Rubio; J. Phys. F: Met. Phys. 14, 1205, (1984).

[12] M. P. L. Sancho, J. M. L. Sancho y J. Rubio; J. Phys. F:Met. Phys. 15, 851-8, (1985).
[13] L. Huang, Y. C. Lai, D. K. Ferry, R. Akis y S. M. Goodnick; J. Phys.: Condens. Matter 21, 344203, (2009).

[14] L. Brey y H. A. Fertig; Phys. Rev. B 73, 235411 (2006).

[15] J. Qi, N. Edirisinghe, M. G. Rabbani y M. P. Anantram; Phys. Rev. B 87, 085404, (2013).

[16] A. Miroshnichenko, S. Flach y Y. S. Kivshar; Rev. Mod. Phys. 82, 02257 (2010). 